\title{
Grand Challenges in Oral Infections and Microbes
}

\author{
Georgios N. Belibasakis* \\ Division of Oral Diseases, Department of Dental Medicine, Karolinska Institutet, Huddinge, Sweden
}

Keywords: oral infections, oral microbes, oral health, oral disease, dentistry, biofilms, oral microbiology and immunology, oral microbial ecology

Oral diseases are widespread among the global population. It is estimated that, as of the middle of the previous decade, 3.5 billion people were living with untreated oral conditions [1]. Apart from the morbidities caused by these diseases on an individual level, they pose a significant public health challenge for national health systems. The most common oral diseases are of microbial etiology, and include dental caries, odontogenic infections, periodontitis, peri-implantitis, and stomatitis [2].

Studies spanning over a century have attempted to decipher the microbes involved in these oral infections by microscopy, culture, and molecular techniques. We now understand that they are largely endogenous polymicrobial infections. In other terms, they are caused by biofilms formed by multiple and varying resident microorganisms that may, under certain conditions, overgrow to act as opportunistic pathogens. We have gathered considerable scientific information on the microbial etiology and pathogenesis of oral infectious diseases, which is often highlighted in specialized fora $[3,4]$. Despite the great scientific progress made in their understanding, oral infections still pose an important prevention and therapeutic challenge for individual patients, dental clinicians, and national health systems alike [2].

Current research trends dictate the use of state-of-the-art high-throughput sequencing technologies for characterizing the oral microbiome and its dysbiotic changes that lead from health and disease [5]. The study of the oral microbiome with open-ended methods allows the discovery or verification of previously unknown, uncultivated, or unsuspected microorganisms in oral infections. It also enables the individual quantification and simultaneous study of interrelationships between microorganisms in a given sample [6]. The term "oral microbiome" is often used interchangeably with "oral microbiota," yet the former implies the collection of genes present in the latter community of microorganisms. The oral microbiome is an integral residential component of the oral cavity and is considered to be the second more diverse regional microbiome of the human body [7]. Stability of the microbiomes results from a dynamic, yet balanced, interaction of microbes with each other, and with their host, in their micro-ecological niche. Environmental pressures that exceed thresholds associated this homeostatic balance will challenge the competitiveness of the resident microbiota leading to dysbiosis and imminent disease. Paradoxically, the diversity of the focal microbiome appears to increase rather than decrease in given oral infections, such as periodontal disease [8-10]. Therefore, measuring the stability of the oral microbiome could be an important utility for clinical prognosis [11] and diagnosis [12]. Achieving the stability linked to a clinically healthy status should be a key consideration for future preventive and therapeutic strategies [13]. Identification of the biological properties that confer this stability is important for preventing the dysbiotic microbial shifts. Acquiring and maintaining a "healthy oral microbiome" is a promising field open for further investigations [14]. Accordingly, for long-term efficacy, research efforts should largely be placed on prevention rather 
than treatment strategies. Our current understanding of oral infections and their associated oral microbiota urges us to reconsider our clinical thinking by embracing more "ecological" approaches for prevention and treatment. In other words, we should strive for preventing or reversing the biological, behavioral, or environmental factors that favor the overgrowth of opportunistic pathogens and the consequent dysbiotic changes that lead to disease [13].

While metagenomics have been instrumental in taxonomically characterizing the oral microbiome, the added dimensions of metatransctriptomics, metaproteomics, and metabolomics enable the classification of the oral microbiota according to biosynthetic, functional, and metabolic properties. These may not necessarily overlap with phylogenetic taxonomy, but the collective assessment of multiple related biological units (genes, gene expression, proteins, metabolites) may enable us to better understand the dynamics of dysbiosis in oral infections. Among the biggest challenges in combined "omics" are data clarity with reduced background noise, reproducibility among different study cohorts, computational integration of different data layers, and meaningful data interpretation for oral disease [6]. Multifaceted data-driven approaches are needed in oral healthcare research. They enable integration of diverse individual characteristics, in order to be able to stratify patients according to individual risk groups and oral healthcare requirements. Using, for instance, microbiological parameters (e.g., stability and dynamics of microbiome or metabolic pathways), adding the patients' risk factors (e.g., genetic, environmental, and behavioral), and channeling the data through supervised and unsupervised learning systems may help identify and manage particularly susceptible individuals or populations. This will customize treatment planning and improve the treatment outcome, moving us away from decisionmaking based on experiential opinion or clinical presentation alone, in line with the concept of precision dentistry $[15,16]$.

The need for personalized monitoring and treatment in dental healthcare is now more evident than ever, and certain facets of our acquired knowledge on the oral microbiome can be well-utilized in this direction [16-18]. Continuously monitoring microbiological parameters, alone or in combination with immunological parameters, in a fast and cost-efficient manner before treatment or during maintenance will facilitate the applications of precision dentistry and personalized

\section{REFERENCES}

1. Kassebaum NJ, Smith AGC, Bernabe E, Fleming TD, Reynolds AE, Vos T, et al. Global, regional, and national prevalence, incidence, and disability-adjusted life years for oral conditions for 195 countries, 1990-2015: a systematic analysis for the global burden of diseases, injuries, and risk factors. J Dent Res. (2017) 96:380-7. doi: 10.1177/0022034517693566

2. Belibasakis GN, Mylonakis E. Oral infections: clinical and biological perspectives. Virulence. (2015) 6:173-6. doi: 10.1080/21505594.2015.1025191

3. Belibasakis GN, Curtis MA, Hajishengallis G, Zaura E. Meeting report: the 12(th) European oral microbiology workshop (EOMW) in Stockholm, Sweden. Virulence. (2018) 9:64-9. doi: 10.1080/21505594.2017. 1376147 oral healthcare. Development of point-of-care devices for microbiological and immunological detection that would assist clinicians in the diagnostic, monitoring, and selection of treatment aspects are underway [19, 20].

One of the most contemporary threats to global health is the advent of antimicrobial resistances. Antibiotics for oral infections are prescribed for prophylactic or therapeutic purposes, but are most often based on professional experience or excessive precaution, rather than scientific evidence, susceptibility tests, or good practice guidelines. Common antibiotic prescriptions in dentistry exceed several other medical and allied health care provider specialties [21] and may not always be deemed necessary [22]. Antibiotics may cause ecological disturbances of the resident microbiota at various sites of the body, even ones distant to the focal infection. They can also induce the selection of resistant strains in the microbiome. Antibioticresistant strains can be found in individuals not recently exposed to antibiotics, and among commensal bacterial species, not only pathogens. This has led to a paradigm shift of how resistances are transferred across the oral microbiome. We now understand that the oral microbiome may act as a general reservoir for the carriage of antibiotic resistances, leading to the concept of the "oral resistome" [23]. In this light, significant research efforts should be placed on preventing or curtailing the unnecessary use of antibiotics for dental purposes, in line with the "One Health" concept that unifies human, public, and environmental health $[24,25]$. In parallel, this raises the need for the discovery and implementation of alternative antimicrobial approaches in dentistry, including manufactured or natural antimicrobial agents [26, 27], photodynamic therapy [28], or concomitant use of adjunctive host modulating agents [29].

A joint forum of communication between basic and clinical research is imperative to address these emerging challenges of the oral domain in the years to come, for the collective benefit of the individual and the population. The Specialty Section of "Oral Infections and Microbes" of Frontiers in Oral Health aspires to become a representative forum for fulfilling this goal.

\section{AUTHOR CONTRIBUTIONS}

The author confirms being the sole contributor of this work and has approved it for publication.
4. Belibasakis GN, Hajishengallis G. Advances in oral mucosal immunity and the microbiome. Adv Exp Med Biol. (2019) 1197:1-9. doi: 10.1007/978-3-030-28524-1_1

5. Bostanci N, Bao K, Greenwood D, Silbereisen A, Belibasakis, G. N. Periodontal disease: from the lenses of light microscopy to the specs of proteomics and next-generation sequencing. Adv Clin Chem. (2019) 93:263-90. doi: 10.1016/bs.acc.2019.07.006

6. Nascimento MM, Zaura E, Mira A, Takahashi N, Ten Cate JM. Second era of OMICS in caries research: moving past the phase of disillusionment. J Dent Res. (2017) 96:733-40. doi: 10.1177/0022034517701902

7. Human Microbiome Project Consortium. Structure, function and diversity of the healthy human microbiome. Nature. (2012) 486:207-14. doi: $10.1038 /$ nature 11234 
8. Dabdoub SM, Tsigarida AA, Kumar PS. Patient-specific analysis of periodontal and peri-implant microbiomes. J Dent Res. (2013) 92:168S-75S. doi: 10.1177/0022034513504950

9. Kumar PS, Mason MR. Mouthguards: does the indigenous microbiome play a role in maintaining oral health? Front Cell Infect Microbiol. (2015) 5:35. doi: 10.3389/fcimb.2015.00035

10. Kilian M, Chapple IL, Hannig M, Marsh PD, Meuric V, Pedersen AM, et al. The oral microbiome-an update for oral healthcare professionals. Br Dent J. (2016) 221:657-66. doi: 10.1038/sj.bdj.2016.865

11. Greenwood D, Afacan B, Emingil G, Bostanci N, Belibasakis GN. Salivary microbiome shifts in response to periodontal treatment outcome. Proteomics Clin Appl. (2020) 14:e2000011. doi: 10.1002/prca.202000011

12. Kumar PS, Leys EJ, Bryk JM, Martinez FJ, Moeschberger ML, Griffen AL. Changes in periodontal health status are associated with bacterial community shifts as assessed by quantitative $16 \mathrm{~S}$ cloning and sequencing. J Clin Microbiol. (2006) 44:3665-73. doi: 10.1128/JCM.00317-06

13. Marsh PD, Zaura E. Dental biofilm: ecological interactions in health and disease. J Clin Periodontol. (2017) 44:S12-22. doi: 10.1111/jcpe.12679

14. Zaura E, Mira A. Editorial: the oral microbiome in an ecological perspective. Front Cell Infect Microbiol. (2015) 5:39. doi: 10.3389/fcimb.2015.00039

15. Agler CS, Moss K, Philips KH, Marchesan JT, Simancas-Pallares M, Beck JD, et al. Biologically defined or biologically informed traits are more heritable than clinically defined ones: the case of oral and dental phenotypes. Adv Exp Med Biol. (2019) 1197:179-89. doi: 10.1007/978-3-030-28524-1_13

16. Beck JD, Philips K, Moss K, Divaris K, Morelli T, Offenbacher S. Advances in precision oral health. Periodontol. (2020) 82:268-85. doi: 10.1111/prd.12314

17. Belibasakis GN, Bostanci N, Marsh PD, Zaura E. Applications of the oral microbiome in personalized dentistry. Arch Oral Biol. (2019) 104:7-12. doi: 10.1016/j.archoralbio.2019.05.023

18. Dawes C, Wong DTW. Role of saliva and salivary diagnostics in the advancement of oral health. J Dent Res. (2019) 98:133-41. doi: 10.1177/0022034518816961

19. Mitsakakis K, Stumpf F, Strohmeier O, Klein V, Mark D, Von Stetten $\mathrm{F}$, et al. Chair/bedside diagnosis of oral and respiratory tract infections, and identification of antibiotic resistances for personalised monitoring and treatment. Stud Health Technol Inform. (2016) 224:61-6.

20. Ozenci V, Rossolini GM. Rapid microbial identification and antimicrobial susceptibility testing to drive better patient care: an evolving scenario. $J$ Antimicrob Chemother. (2019) 74:i2-5. doi: 10.1093/jac/dky529
21. Durkin MJ, Hsueh K, Sallah YH, Feng Q, Jafarzadeh SR, Munshi KD, et al. An evaluation of dental antibiotic prescribing practices in the United States. J Am Dent Assoc. (2017) 148:878-86 e1. doi: 10.1016/j.adaj.2017.07.019

22. Swift JQ, Gulden WS. Antibiotic therapy-managing odontogenic infections. Dent Clin North Am. (2002) 46:623-33, vii. doi: 10.1016/s0011-8532(02)00031-9

23. Sukumar S, Roberts AP, Martin FE, Adler CJ. Metagenomic insights into transferable antibiotic resistance in oral bacteria. J Dent Res. (2016) 95:969-76. doi: 10.1177/0022034516648944

24. Ryu S, Kim BI, Lim JS, Tan CS, Chun BC. One health perspectives on emerging public health threats. J Prev Med Public Health. (2017) 50:411-4. doi: 10.3961/jpmph.17.097

25. McEwen SA, Collignon PJ. Antimicrobial resistance: a one health perspective. Microbiol. Spectr. (2018) 6. doi: 10.1128/microbiolspec.ARBA-0009-2017

26. Allaker RP, Ian Douglas CW. Non-conventional therapeutics for oral infections. Virulence. (2015) 6:196-207. doi: 10.4161/21505594.2014. 983783

27. Cao Y, Naseri M, He Y, Xu C, Walsh LJ, Ziora ZM. Non-antibiotic antimicrobial agents to combat biofilm-forming bacteria. J Glob Antimicrob Resist. (2019). doi: 10.1016/j.jgar.2019.11.012. [Epub ahead of print].

28. Cieplik F, Deng D, Crielaard W, Buchalla W, Hellwig E, Al-Ahmad A, et al. Antimicrobial photodynamic therapy-what we know and what we don't. Crit Rev Microbiol. (2018) 44:571-89. doi: 10.1080/1040841X.2018. 1467876

29. Donos N, Calciolari E, Brusselaers N, Goldoni M, Bostanci N, Belibasakis GN. The adjunctive use of host modulators in non-surgical periodontal therapy. A systematic review of randomized, placebo-controlled clinical studies. J Clin Periodontol. (2019). doi: 10.1111/jcpe.13232. [Epub ahead of print].

Conflict of Interest: The author declares that the research was conducted in the absence of any commercial or financial relationships that could be construed as a potential conflict of interest.

Copyright (C) 2020 Belibasakis. This is an open-access article distributed under the terms of the Creative Commons Attribution License (CC BY). The use, distribution or reproduction in other forums is permitted, provided the original author(s) and the copyright owner(s) are credited and that the original publication in this journal is cited, in accordance with accepted academic practice. No use, distribution or reproduction is permitted which does not comply with these terms. 